\title{
Muscle Power during Standing and Seated Trunk Rotations with Different Weights
}

\author{
Erika Zemková \\ Comenius University in Bratislava, Department of Sports Kinanthropology, Faculty of Physical Education and Sport, Bratislava, \\ Slovakia \\ Slovak University of Technology, Sports Technology Institute, Faculty of Electrical Engineering and Information Technology, \\ Bratislava, Slovakia \\ Michal Jeleň \\ Slovak University of Technology, Sports Technology Institute, Faculty of Electrical Engineering and Information Technology, \\ Bratislava, Slovakia \\ Ludmila Zapletalová \\ Comenius University in Bratislava, Department of Sports Games, Faculty of Physical Education and Sport, Bratislava, Slovakia \\ Dusan Hamar \\ Comenius University in Bratislava, Department of Sports Kinanthropology, Faculty of Physical Education and Sport, \\ Bratislava, Slovakia
}

\begin{abstract}
A B S T R A C T
This study compares peak and mean power during standing and seated trunk rotations with different weights. Twenty seven fit men completed four trials of trunk rotations in both standing and seated positions with a bar weight of $5.5,10.5,15.5$, and $20 \mathrm{~kg}$ placed on the shoulders. The FiTRO Torso Premium was used to monitor basic biomechanical parameters throughout the movement. Results showed significantly higher peak power during standing than seated trunk rotations at weights of $20 \mathrm{~kg}$ (274.4 \pm 63.5 vs. $206.4 \pm 54.6 \mathrm{~W}, p=0.004), 15.5 \mathrm{~kg}(371.2 \pm 93.9 v s .313 .5 \pm 72.3 \mathrm{~W}, p=0.007)$, and $10.5 \mathrm{~kg}(336.9 \pm 77.8 v s .286 .3 \pm 66.0$ $W, p=0.009)$ but not at $5.5 \mathrm{~kg}(191.6 \pm 46.2 \mathrm{vs} .166 .0 \pm 37.0 \mathrm{~W}, p=0.061)$. Similarly, mean power in the acceleration phase of trunk rotations was significantly higher when performed in standing than seated position at weights of $20 \mathrm{~kg}(143.2 \pm 32.1 \mathrm{vs} .101 .9 \pm 23.7$ $W, p=0.008), 15.5 \mathrm{~kg}(185.1 \pm 42.3 \mathrm{vs} .150 .4 \pm 36.5 \mathrm{~W}, p=0.019)$, and $10.5 \mathrm{~kg}(169.8 \pm 40.7 \mathrm{vs} .139 .7 \pm 31.6 \mathrm{~W}, p=0.024)$ but not at 5.5 $\mathrm{kg}(107.4 \pm 29.4$ vs. $86.5 \pm 21.1 \mathrm{~W}, p=0.111)$. Furthermore, peak and mean power during standing trunk rotations significantly correlated with values achieved in the seated position at the weight of $5.5 \mathrm{~kg}(r=0.684, p=0.027 ; r=0.676, p=0.033)$ but not at 10.5 $\mathrm{kg}(r=0.589, p=0.089 ; r=0.552, p=0.143), 15.5 \mathrm{~kg}(r=0.493, p=0.243 ; r=0.436, p=0.298)$, and $20 \mathrm{~kg}(r=0.357, p=0.361 ; r=0.333$, $p=0.417)$. In conclusion, power production is greater during standing as compared to seated trunk rotations, with more pronounced differences at higher weights. This fact has to be taken into account when training and testing the trunk rotational power.
\end{abstract}

Key words: additional load, rotational power, standing/sitting positions, trunk movement

\section{Introduction}

There is considerable debate on effectiveness of seated vs standing resistance exercises. Though seated exercises are more stable and safer, these seems to be less effective in power production than standing resistance exercises. This is especially true for rotational movements of the torso when using an additional load. If the weight is heavy, the use of legs can help to execute the exercise more effectively although maintaining balance may be somewhat more difficult during standing than seated trunk rotations.

The same issue may be observed when testing muscle strength and power during trunk rotational movements. Typically, isokinetic machines (Newton, Thow, Somerville, Henderson, \& Waddell, 1993; Kumar, Dufresne, \& Van Schoor, 1995; Kumar, 1997) or electromyography (Pope, Andersson, Broman, Svensson, \& Zetterberg, 1986; McGill, 1991; Kumar \& Narayan, 1998) are used to measure strength characteristics during axial rotation movements. However, when using an isokinetic dynamometer with a torso rotation attachment, no significant differences in peak torque were found within or between groups of healthy individuals who do not play golf and those who are highly skilled at the sport (Lindsay \& Horton, 2006). The authors also reported no significant difference in the endurance of trunk muscles between the healthy elite golfers and the nongolfing controls. Similarly, Suter and Lindsay (2001) were unable to show any significant differences in the static holding times or a decline in the electromyography median frequency between low-handicap golfers with low back pain and healthy, age-matched controls who did not golf.

The limitation of these measurements is that torso rotation performed while sitting on the chair with straps around the back and legs provides artificial movement patterns. Additionally, most of the custom-made equipments are relatively expensive and not portable for use on the sporting field. To avoid these drawbacks, one can use a system attached to the barbell or weight machine that allows monitoring of basic biomechanical parameters during rotational movement of the trunk. So far, the 
study of Andre et al. (2012) determined the test-retest reliability of the kinetic rotational characteristics of the pulley trainer when performing a rotational exercise of the axial skeleton in the transverse plane while sitting on a box. The authors found that a pulley system and an external dynamometer can be used together as a reliable research tool to assess rotational power (ICC values above 0.90). Although such a test is suitable for canoeing, for example, for many other sports, such as hockey or tennis, rotational movement performed during standing would be a more specific alternative.

Therefore, the exercise that closely replicates the upper/lower body rotation movements should be preferred in testing in order to assess sport-specific power. The test adapted from the wood chop exercise provides conditions similar to those imposed in many sports involving trunk rotation in standing position (baseball, golf, karate, etc.). Recent study showed that evaluation of maximal muscle power and trunk rotational endurance during the standing cable wood chop exercise on a weight stack machine is both a reliable method and sensitive to differences among physically active individuals (Zemková, Cepková, Uvaček, \& Šooš, 2016). Specifically, mean rotational power is reliable (ICC values above 0.90 at all weights tested) and sensitive parameter able to discriminate within-group differences. Therefore, this method of assessing (a) maximal values of power using maximal effort single repetitions of the standing cable wood chop exercise with increasing weights and (b) the rotational endurance of the trunk muscles using a set of a predetermined number of repetitions performed at a previously established weight at which maximal power was achieved may be used in functional performance testing, namely for athletes who require the production of rotational power during training or competition.

Such a computer-based system that can be directly connected to the weights on stack machine may be considered to be a suitable and practical alternative for sport-specific and fitnessoriented testing of trunk rotational power. However, some practitioners prefer free weights in their weight training workout routine. While machines are good for training of muscle strength they neglect key stabilization components of the core. Using free weights is a way to 'functional' training that places greater demands on stabilizing muscles. In addition, exercises with free weights allow performing a full range of trunk motion. Moreover, free-weight exercises are closer to many sports and daily activities, can be performed in any sporting fields and are less expensive than exercises on weight machines.

Accordingly, it is necessary to provide testing conditions as close as possible to those used during training and/or competition. Usually, single repetitions of a particular exercise with increasing weights stepwise up to the 1 repetition maximum are performed to obtain individual force-velocity and power-velocity curves or to analyze power/velocity and weight lifted relationship. It is known that with increasing weights there is a decrease in velocity in the concentric phase of lifting. Contrary to this, power increases from lower weights, reaches a peak, and then toward higher weights, decreases again. Such an optimal velocity, that is, the one allowing the production of the greatest power, depends on ratio of fast and slow twitch muscle fibers (Tihanyi, Apor, \& Fekete, 1982); thus, it may be hardly changed with training. However, the optimal weight at which maximal power is achieved may increase with the training.

This testing procedure consisting of maximal effort single repetitions with increasing weights may also be applied for obtaining maximal values of trunk rotational power. Since standing rotational movement allowing more involvement of the lower body is less confined to the trunk, it is likely that it is much more effective in power production than seated trunk rotations. However, the question remains to what extent the rotational power during standing and sitting depends on amount of the load used. This study aims to test the hypothesis that differences in peak and mean values of power between standing and seated trunk rotations are more pronounced at higher than lower weights.

\section{Methods}

\section{Participants}

Twenty seven fit men (age $22.7 \pm 2.9$ y, height $178.6 \pm 8.6$ $\mathrm{cm}$, body mass $86.3 \pm 11.1 \mathrm{~kg}$ ) volunteered to participate in the study. All of them had on average 3.5 years' experience with resistance training including exercises to strenghten the trunk muscles. They were no active in any sport at a competitive level that would require loaded trunk rotations. The participants were included in the study only if they subjectively did not report back pain. Individuals who had previously undergone surgery or other medically invasive procedures for low back pain were

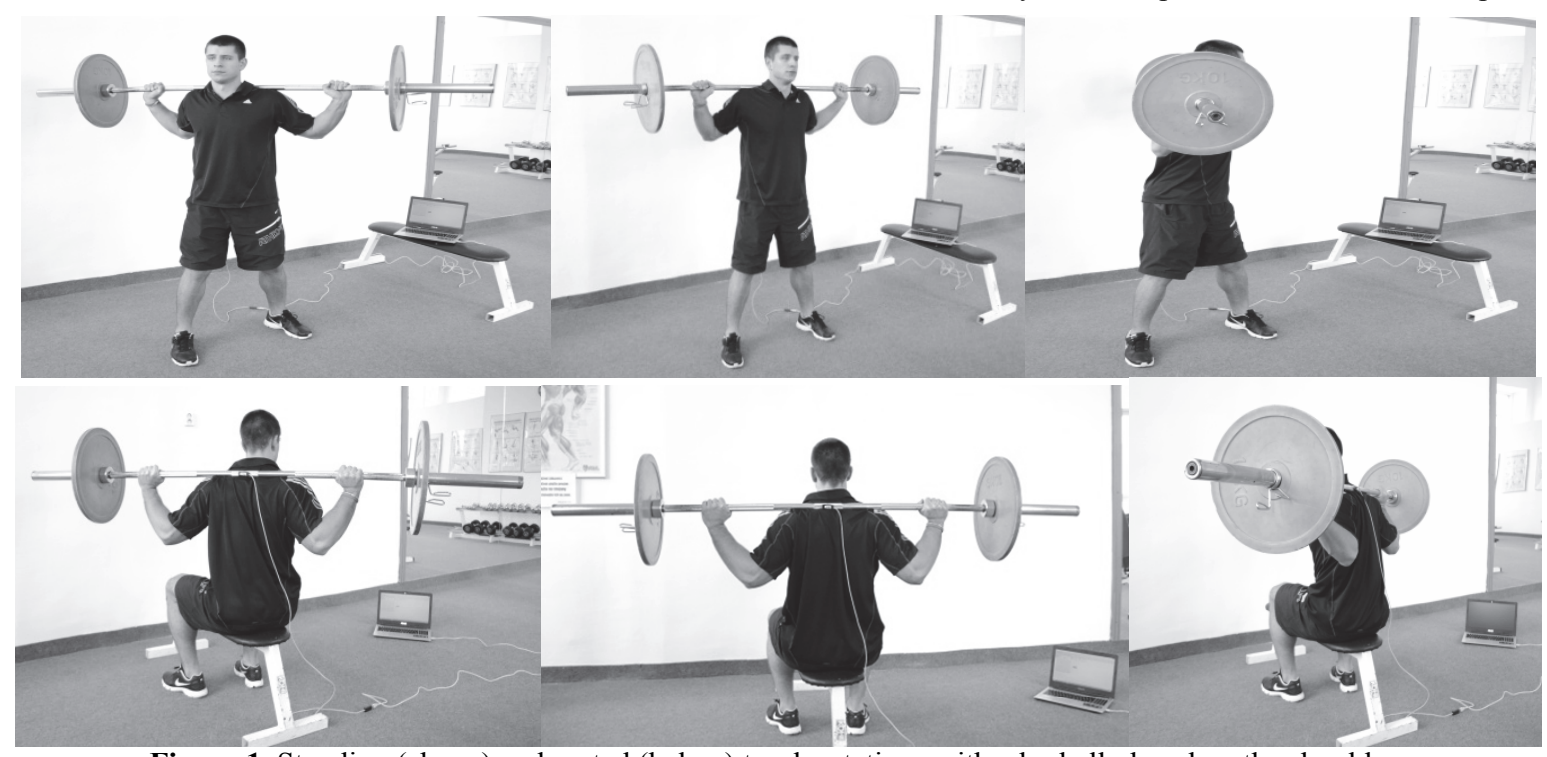

Figure 1. Standing (above) and seated (below) trunk rotations with a barbell placed on the shoulders 
excluded from participation in the study. All of them were informed of the procedures and the main purpose of the study. The procedures presented were in accordance with the ethical standards on human experimentation and in compliance with the Helsinki Declaration.

\section{Experimental Protocol}

Participants were asked to avoid any strenuous exercises during the study. The procedure and time of day were identical for all participants. The same experienced researchers conducted the measurements during testing sessions.

Following the warm-up, participants were exposed to a familiarization trial during which they performed standing and seated trunk rotations in a slow and controlled manner while keeping the back straight. Afterwards, they completed two repetitions of trunk rotations to each sides in both standing and seated positions with a bar weight of $5.5,10.5,15.5$, and $20 \mathrm{~kg}$ placed on the shoulders (Figure 1). They were instructed to perform trunk rotations with maximal effort in the acceleration phase. Emphasis was placed on the proper position of the body, namely during the standing trunk rotations. In the first case they stood with their feet wider than shoulder width apart and toes slightly pointed outwards, in the second they seated on a bench with feet placed on the ground while holding the barbell on the shoulders. They rotated their torso forcefully from the right (or the left) towards the opposite side until the body reached the end position, and then they slowly returned to the starting position. The test was then repeated for the opposite side of the body. They had to engage their abdominal / core muscles to stiffen the torso and stabilize the spine. A laboratory assistant made sure that participants remained upright throughout the movement and that their head, chest and torso were aligned over their hips.

\section{Power Assessment}

Basic biomechanical parameters throughout the movement were monitored using the FiTRO Torso Premium system (FiTRONiC, Slovakia), Figure 2. The system consists of an inertia measurement unit in a small box with an integrated USB interface and software. While inserted on the barbell axis, the sensor unit registers instant angular of rotation movement. Calculations of force and power are based on the Newton's second law of mechanics. Force produced to accelerate and decelerate a rotation movement is obtained as a product of barbell mass and acceleration of its center of gravity $(\mathrm{CoG})$. Angular acceleration is obtained by derivation of angular velocity. For the transformation of angular velocity and acceleration into their real values, a rotation radius (distance between rotation axis and barbell mass $\mathrm{CoG}$ ) is used. Power is calculated as a product of force and velocity.
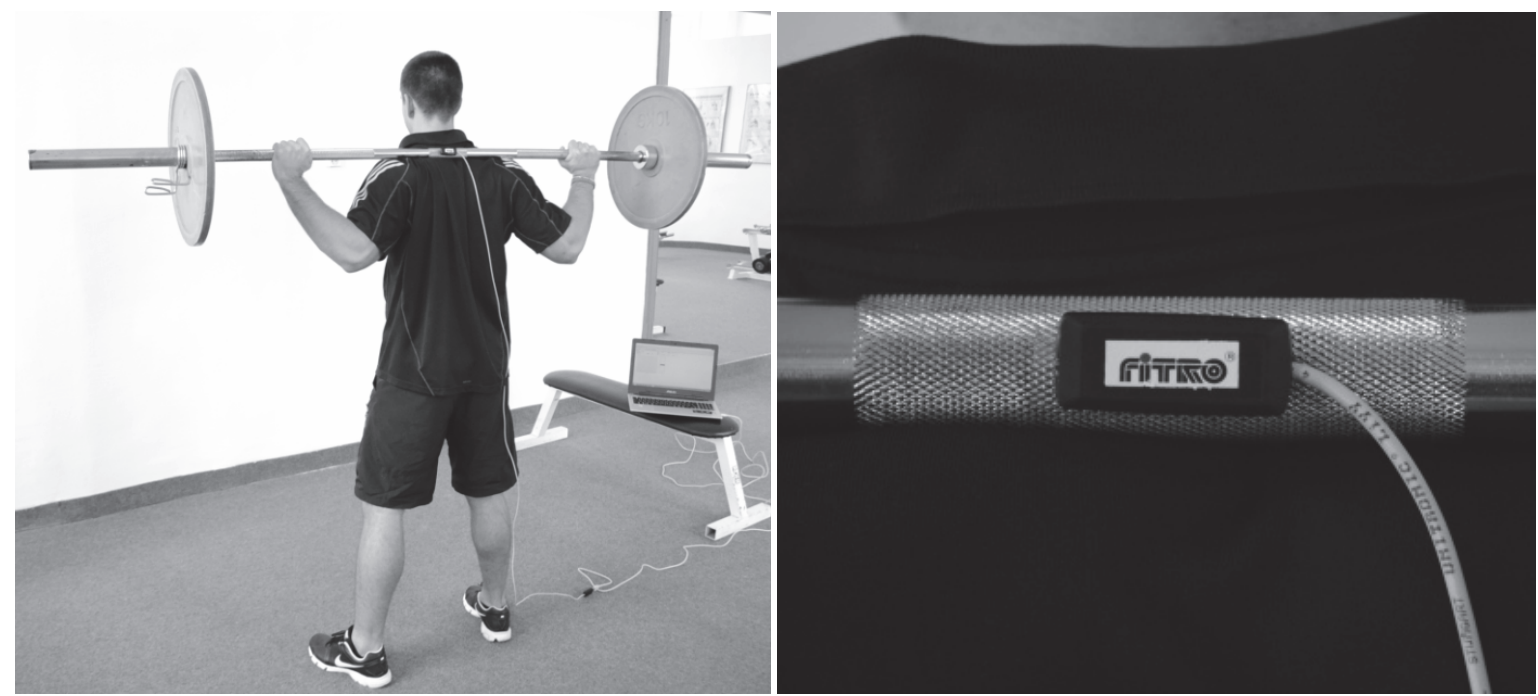

Figure 2. Measurement of basic biomechanical parameters throughout the trunk rotational movement (left) using the FiTRO Torso Premium system (right)

\section{Statistical Analysis}

Data analyses were performed using the statistical program SPSS for Windows version 18.0 (SPSS, Inc., Chicago, IL, USA). Analysis of variance (ANOVA) with repeated measures design was used to determine the differences in peak and mean values of power for two types of trunk rotations (standing, seated) at four loading conditions $(5.5,10.5,15.5$, and $20 \mathrm{~kg})$. Bonferroni's post hoc analysis was conducted if the ANOVA showed statistically significant main effects or interaction effects. The significance level was set at $p<0.05$. The relationship between trunk rotational power in the standing and seated position with all weights used was established using Pearson's product moment correlation coefficient. All data were presented as means and standard deviations.

Previous study showed no significant differences in peak torque in strength testing or in total work in rotational endurance testing between the dominant and non-dominant side in healthy golfers, control group and golfers with low back pain
(Lindsay \& Horton, 2006). Likewise, there were no significant differences in mean power produced during the standing cable wood chop exercise on the left and the right side with all weights used in a group of fit healthy men (Zemková et al., 2016). Therefore, we assumed no side-to-side differences in power outputs in participants of the present study and used average values of better trial on each side for data analysis.

\section{Results}

The ANOVA revealed a significant effect for the type of trunk rotations on peak and mean power $(F=11.76, P=0.002$ and $F=10.73, P=0.003$, respectively). The ANOVA also identified a significant main effect for loading conditions on peak and mean power $(F=8.24, P=0.004$ and $F=7.76, P=0.005$, respectively). In addition, there was a significant type of trunk rotations $\mathrm{x}$ loading conditions interaction for peak and mean values 
of power $(F=7.17, P=0.007$ and $F=6.39, P=0.009$, respectively).

Peak power was significantly higher during standing than seated trunk rotations at weights of $20 \mathrm{~kg}(24.8 \%, p=0.004)$, $15.5 \mathrm{~kg}(15.5 \%, p=0.007)$, and $10.5 \mathrm{~kg}(15.0 \%, p=0.009)$ but not at lower weight of $5.5 \mathrm{~kg}(13.4 \%, p=0.061)$ (Figure 3).

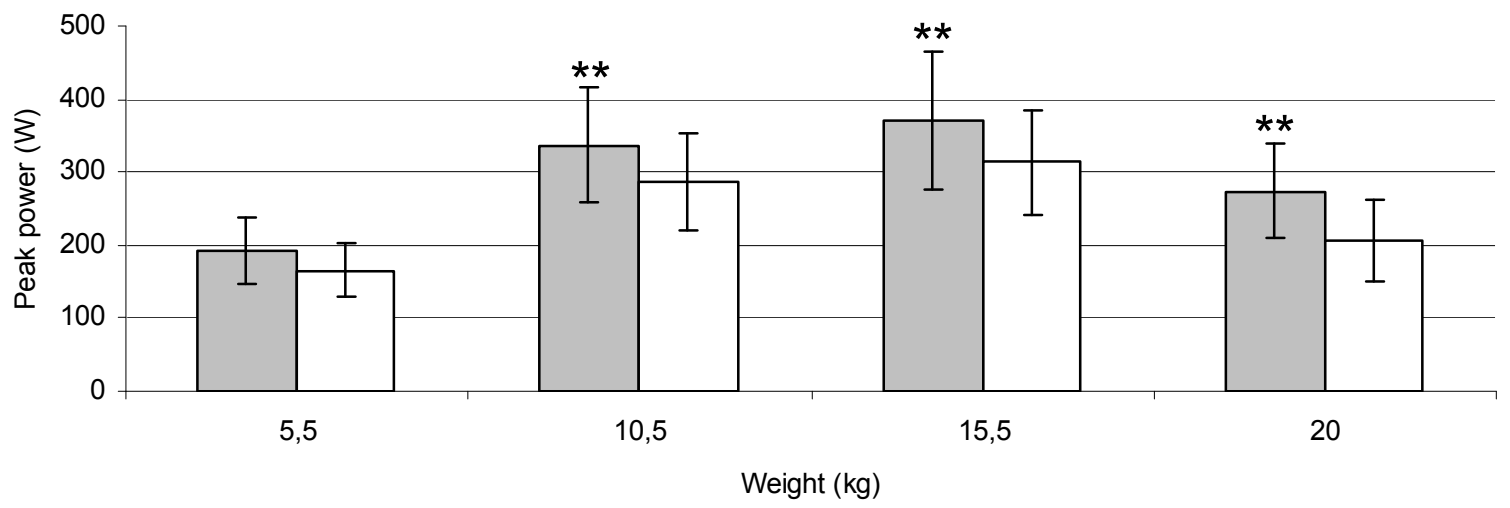

$\square$ Standing trunk rotations $\square$ Seated trunk rotations

Figure 3. Peak power during standing and seated trunk rotations with different weights $(* * p \leq 0.01)$

Mean power in the acceleration phase of trunk rotations was also significantly higher in standing than seated position at weights of $20 \mathrm{~kg}(28.8 \%, p=0.008), 15.5 \mathrm{~kg}(18.7 \%, p=0.019)$, and $10.5 \mathrm{~kg}(17.7 \%, p=0.024)$ but not at $5.5 \mathrm{~kg}(19.5 \%$, $p=0.111$ ) (Figure 4).

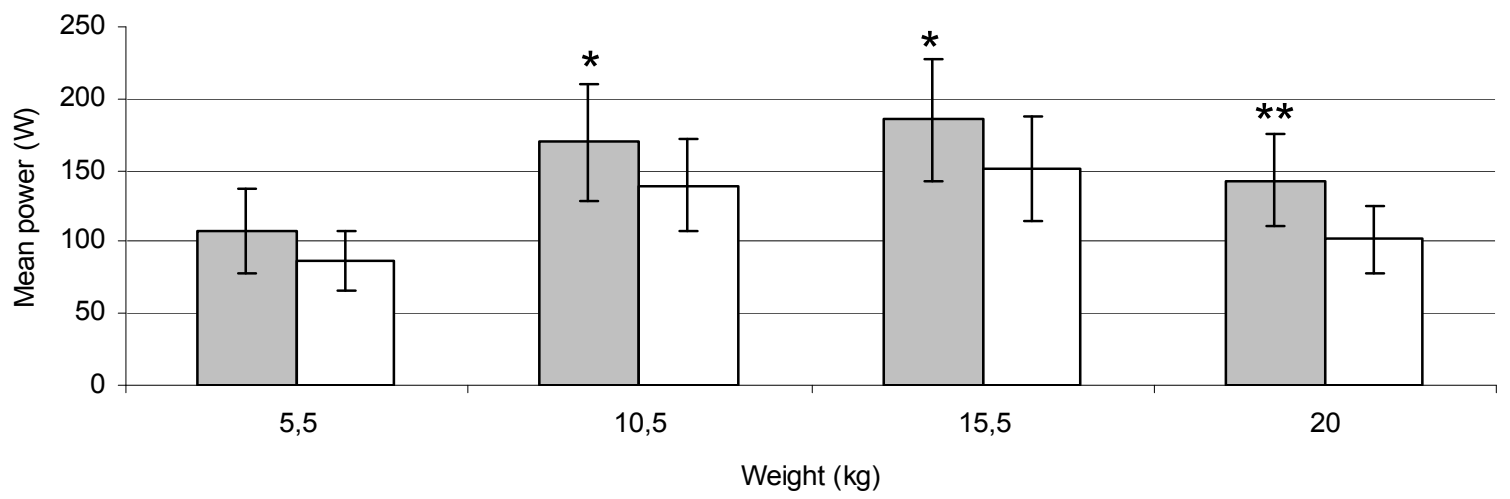

$\square$ Standing trunk rotations $\square$ Seated trunk rotations

Figure 4. Mean power in the acceleration phase of standing and seated trunk rotations with different weights $\left({ }^{*} p \leq 0.05,{ }^{* *} p \leq 0.01\right)$

Differences in peak and mean values of trunk rotational and 6. power while standing and sitting are displayed in Figures 5

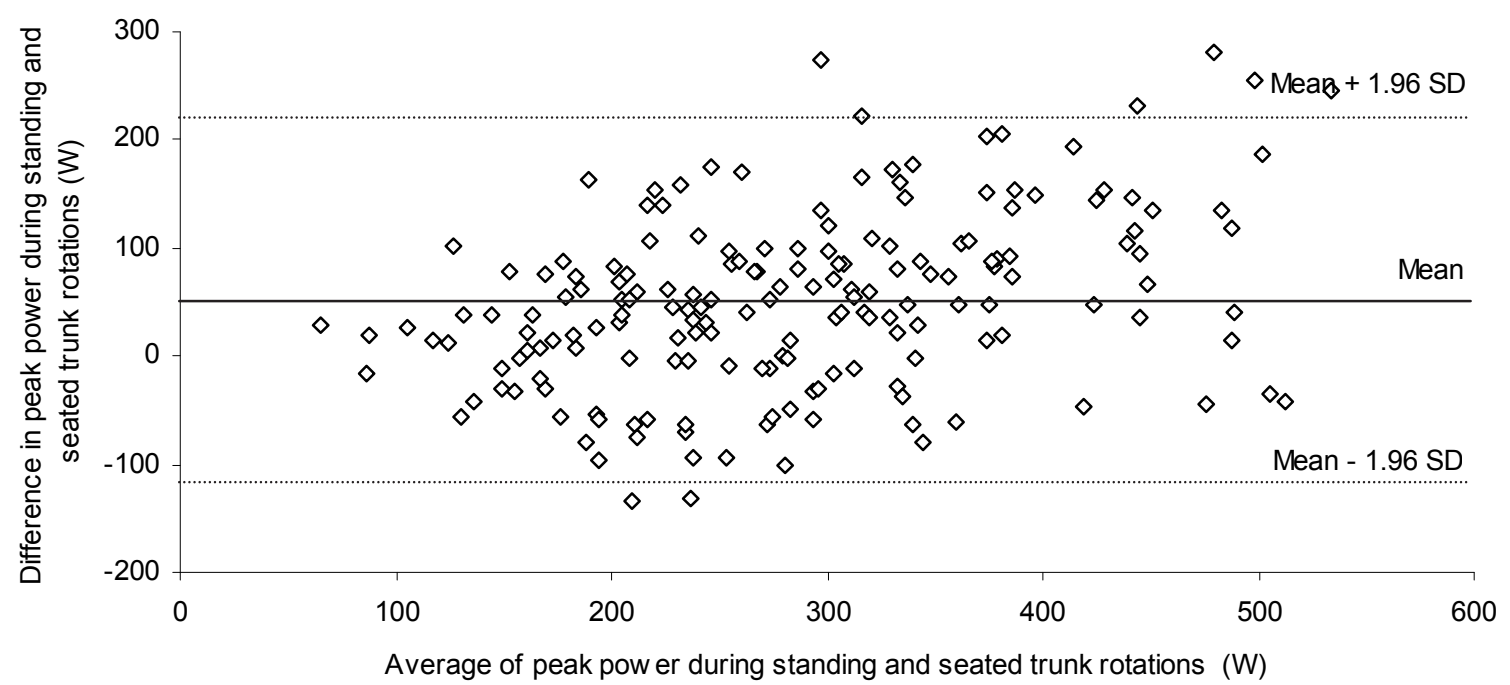

Figure 5. The difference in peak power during standing and seated trunk rotations 
Furthermore, there were significant correlations between peak and mean values of power during standing and seated trunk rotations with the weight of $5.5 \mathrm{~kg}(\mathrm{r}=0.684, p=0.027$, $95 \%$ CI $[0.621,0.712] ; \mathrm{r}=0.676, p=0.033,95 \%$ CI $[0.617$, $0.707])$, whereas low correlations were found at weights of 10.5 $\mathrm{kg} \quad(\mathrm{r}=0.589, \quad p=0.089,95 \%$ CI $[0.542,0.619] ; \quad \mathrm{r}=0.552$, $p=0.143,95 \%$ CI $[0.501,0.586]), 15.5 \mathrm{~kg}(\mathrm{r}=0.493, p=0.243$, $95 \%$ CI [0.442, 0.523$] ; \mathrm{r}=0.436, p=0.298,95 \%$ CI [0.390, $0.463])$, and $20 \mathrm{~kg}(\mathrm{r}=0.357, p=0.361,95 \% \mathrm{CI}[0.313,0.393]$; $\mathrm{r}=0.333, p=0.417,95 \%$ CI $[0.294,0.366])$.

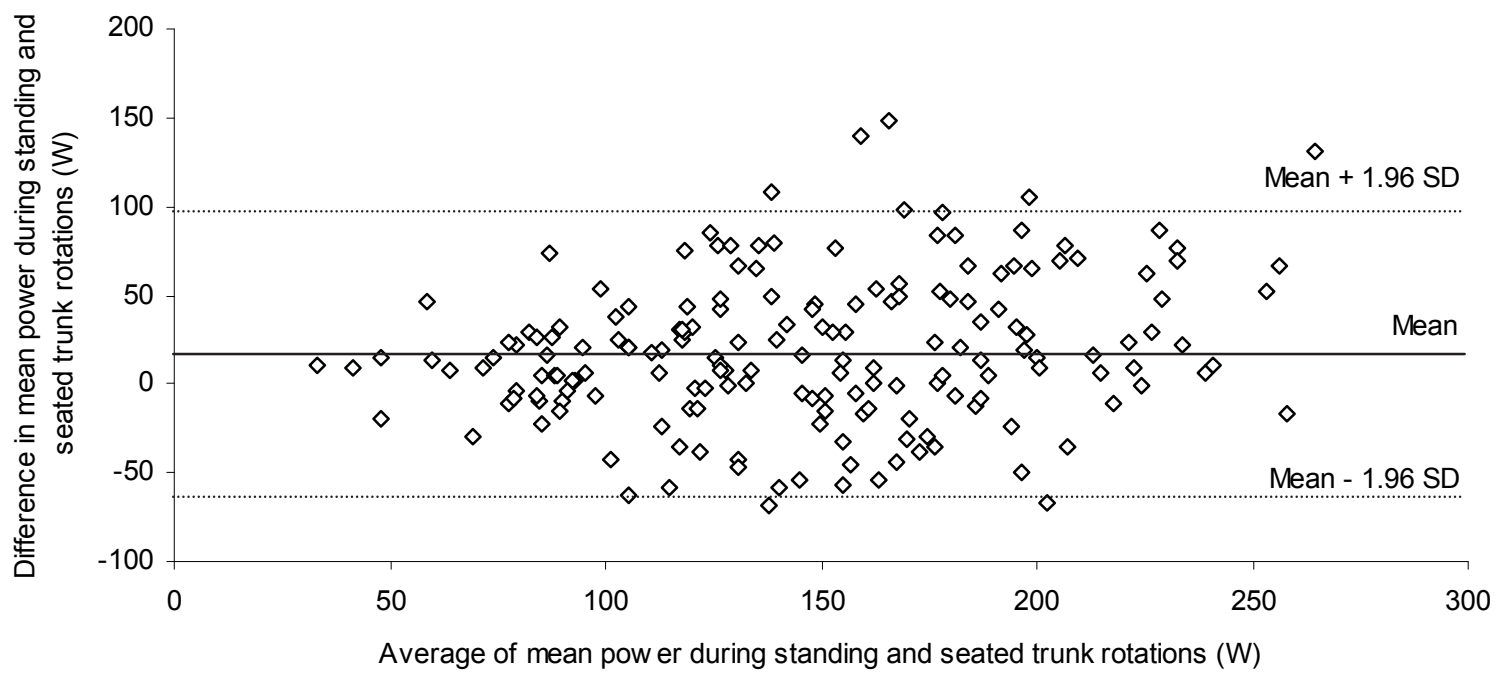

Figure 6. The difference in mean power in the acceleration phase of standing and seated trunk rotations

\section{Discussion}

Peak and mean values of power were significantly higher during standing than seated trunk rotations, however only with weights $\geq 10.5 \mathrm{~kg}$. This may be ascribed to a greater range of trunk motion while standing as compared to sitting, which allowed participants to accelerate the movement more forcefully at the beginning of rotation. As a result was a greater trunk rotational velocity and consequently also overall power outputs. Indeed, peak and mean values of velocity in the acceleration phase of trunk rotation as well as respective angular displacements were significantly higher during standing than seated trunk rotations with weights of $20,15.5$, and $10.5 \mathrm{~kg}$ but with the weight of $5.5 \mathrm{~kg}$.

On the other hand, seated trunk rotations reduced the involvement of legs and contribution of thoracic/hip mobility to the upper-body rotational power. Reduced range of motion of the hips and the thoracic spine, which allow the greatest rotation because of the orientation of the joints (Sahrmann, 2002), could contribute to lower movement velocity of the trunk and consequently influence ball velocity in throwing and striking sports. These sports that involve throwing motions require production of explosive movement in either the transverse or oblique planes (Earp \& Kraemer, 2010). The force is transferred sequentially from the proximal segments, such as hips, toward the more distal segments, such as the shoulders and arms. Because of the kinetic linkage of the proximal to distal sequence in throwing (Putnam, 1993), the rotational mobility may play an important role in production of trunk rotational power. This power transference of the proximal segments, such as the hips and upper trunk, may be crucial to throwing velocity.

Core strength does have a significant effect on an athlete's ability to create and transfer forces to the extremities (Shinkle, Nesser, Demchak, \& McMannus, 2012). It is obvious that the effective execution of the tennis stroke or golf swing not only requires rapid movement of the extremities but also substantial rotational power and/or velocity of the trunk muscles. Trunk extensors, flexors, rotators, and lateral bend agonists are active throughout the stroke in baseball and tennis as well as the golf swing. Actually, all trunk muscles are relatively active during the acceleration phase of the golf swing with the trail-side abdominal oblique muscles showing the highest level of activity (Watkins, Uppal, Perry, Pink, \& Dinsay, 1996).

Given the importance of trunk rotational power in these sports, core strengthening and core stabilization exercises should be considered an integral part of functional training. Core exercises incorporated into strength and power training regimens require bilateral agonist-antagonist coactivation to produce movement and stabilize the spine. When the trunk muscles must be co-activated to stabilize the spine, that exercise is by definition a core stability exercise (Lehman, 2006). Core stability is the ability of the lumbopelvic-hip structures and musculature to withstand compressive forces on the spine and return the body to equilibrium after perturbation (Willson, Dougherty, Ireland, \& Davis, 2005). Factors such as the endurance, strength, power, and coordination of the abdominal, hip, and spine musculature are important components of core stability. The study by Keogh, Aickin, \& Oldham (2010) suggests that similar to strength, core stability exhibits relatively high levels of task specificity. The implication of this is that once some initial conditioning of the core musculature is obtained, core stability training should be as specific as other aspects of the conditioning program if functional performance is to be improved. It could be argued that one way to achieve this would be the use of functional total body exercises that mimic in some respects actual movements that are routinely performed by the athletes in their sports. These total body exercises may also be used to assess functional core stability. The challenge remains as to what aspects of performance in these total body tasks would be assessed and how this would be quantified in an objective manner.

Selecting a single appropriate test to fully evaluate core stability is difficult, given the complex interaction of the lumbopelvic-hip structures and musculature. A number of static single-joint core stability measures and ratios were unable to 
distinguish resistance-trained subjects with high and low strength and power levels or to evaluate the efficiency of training involving complex dynamic core exercises. Implements, such as the medicine ball and cable pulleys, can be very useful in developing and quantifying power as they allow motion in all three planes. Both medicine ball throws (side, overhead, scoop) and the chop and lift for rotational power assessment have shown high reliability (ICC $=0.84-0.99$ and $0.87-0.98$, respectively) (Kohmura, Aoki, Yoshigi, Sakuraba, \& Yanagiya, 2008; Palmer \& Uhl, 2011; Rivilla-García, Martínez, Grande, \& Sampedro-Molinuevo, 2011; Lehman, Drinkwater, \& Behm, 2013). Rivilla-Garcia et al. (2011) reported a high correlation $(\mathrm{r}=0.90)$ between a light overhead medicine ball throw $(0.8 \mathrm{~kg})$ and handball-throwing velocity. Conversely, Kohmura et al. (2008) reported that the scoop medicine ball throw has very little shared variance with baseball fielding (throwing distance, standing long jump, and agility T-test) $(\sim 7 \%)$ compared with batting ( 14\%). Recently, Talukdar, Cronin, Zois, and Sharp (2015) examined the role of rotational power and mobility on cricet ball throwing velocity using a linear position transducer attached to the weight stack of a cable pulley system to measure chop and lift power. According to the authors, greater ROM at proximal segments, such as hips and thoracic, may not increase throwing velocity in cricet as reduced ROM at proximal segments can be useful in transfering the momentum from the lower extremity in an explosive task such as throwing. These discrepancies may be ascribed to the task specificity and weight of the medicine ball or amount of load used during the chop and lift.

Thus, there is a need to develop a sport-specific test able to evaluate rotational power of the trunk. It is especially important to design the test that require little or no equipment and hence is inexpensive and fast to administer. Most current tests evaluate the endurance (e.g., trunk flexor and extensor endurance tests and the lateral bridge test) rather than the strength and power component of core stability. Given that rotational power is a

\section{R E F E R E N C E S}

Andre, M.J., Fry, A.C., Heyrman, M.A., Hudy, A., Holt, B., Roberts, C., Vardiman, J.P., \& Gallagher, P.M. (2012). A reliable method for assessing rotational power. Journal of Strength and Conditioning Research, 26(3), 720-724.

Earp, J.E. \& Kraemer, W.J. (2010). Medicine ball training implications for rotational power sports. Strength and Conditioning Journal, 32(4), 20-25.

Keogh, J.W., Aickin, S.E., \& Oldham, A.R. (2010). Can common measures of core stability distinguish performance in a shoulder pressing task under stable and unstable conditions? Journal of Strength and Conditioning Research, 24(2), 422-429.

Kohmura, Y., Aoki, K., Yoshigi, H., Sakuraba, K., \& Yanagiya, T. (2008). Development of a baseball-specific battery of tests and a testing protocol for college baseball players. Journal of Strength and Conditioning Research, 22(4), $1051-1058$.

Kumar, S., Dufresne, R.M., \& Van Schoor, T. (1995). Human trunk strength profile in lateral flexion and axial rotation. Spine, 20(2), 169-177.

Kumar, S. (1997). Axial rotation strength in seated neutral and pre-rotated postures of young adults. Spine, 22(19), 22132221.

Kumar, S. \& Narayan, Y. (1998). Spectral parameters of trunk muscles during fatiguing isometric axial rotation in neutral better predictor of athlete performance, the test that measure this component of the core may be more useful, especially because it may better mimic the demands imposed by sports. The value of this novel method for assessing muscle power during the standing or seated trunk rotations is that it simulates movement that occurs in many sports (baseball, basketball, cricket, golf, hockey, tennis, soccer, canoeing etc.). The difference is mainly the velocity of the trunk movement and the unloading or loading conditions under which it is being performed.

In the present study, low correlations between the power achieved during standing and seated trunk rotations with weights $\geq 10.5 \mathrm{~kg}$ were found, suggesting that these tests measure distinct qualities. This is because core muscles facilitate the movement of the trunk easier when the body is in an upright position. On the contrary, there was a strong relationship between power produced during standing and seated trunk rotations with lower weight of $5.5 \mathrm{~kg}$. This indicates that these exercises are similar in terms of power production.

In conclusion, muscle power is greater during standing as compared to seated trunk rotations, with more pronounced differences at higher weights $(\geq 10.5 \mathrm{~kg})$. These findings indicate that standing trunk rotations are much more effective for power production than those performed in the seated position. Therefore, in sports involving loaded trunk rotations, standing posture should be preferred when testing athlete's specific performance as opposite to currently used dynamometers allowing movements of the trunk in seated and fixed position.

\section{Acknowledgement}

This work was supported by the Scientific Grant Agency of the Ministry of Education, Science, Research and Sport of the Slovak Republic and the Slovak Academy of Sciences (No. 1/0824/17) and the Slovak Research and Development Agency under the contract No. APVV-15-0704.

posture. Journal of Electromyography and Kinesiology, $8(4), 257-267$.

Lehman, G.J. (2006). Resistance training for performance and injury prevention in golf. The Journal of the Canadian Chiropractic Association, 50(1), 27-42.

Lehman, G., Drinkwater, E.J., \& Behm, D.G. (2013). Correlation of throwing velocity to the results of lower-body field tests in male college baseball players. Journal of Strength and Conditioning Research, 27(4):902-908.

Lindsay, D.M. \& Horton, J.F. (2006). Trunk rotation strength and endurance in healthy normals and elite male golfers with and without low back pain. North American Journal of Sports Physical Therapy, 1(2), 80-88.

McGill, S.M. (1991). Electromyographic activity of the abdominal and low back musculature during the generation of isometric and dynamic axial trunk torque: Implications for lumbar mechanics. Journal of Orthopaedic Research, 9(1), 91-103.

Newton, M., Thow, M., Somerville, D., Henderson, I., \& Waddell, G. (1993). Trunk strength testing with iso-machines. Part 2: Experimental evaluation of the Cybex II Back Testing System in normal subjects and patients with chronic low back pain. Spine, 18(7), 812-824.

Palmer, T.G., \& Uhl, T.L. (2011). Interday reliability of peak muscular power outputs on an isotonic dynamometer and 
assessment of active trunk control using the chop and lift tests. Journal of Athletic Training, 46(2):150-159.

Pope, M.H., Andersson, G.B., Broman, H., Svensson, M., \& Zetterberg, C. (1986). Electromyographic studies of the lumbar trunk musculature during the development of axial torques. Journal of Orthopaedic Research, 4(3), 288-297.

Putnam, C.A. (1993). Sequential motions of body segments in striking and throwing skills: descriptions and explanations. Journal of Biomechanics, 26(Suppl 1), 125-135.

Rivilla-García, J., Martínez I, Grande, I., \& SampedroMolinuevo, J. (2011). Relation between general throwing tests with a medicine ball and specific tests to evaluate throwing velocity with and without opposition in handball. Journal of Human Sport \& Exercise, 6(2), 414-426.

Sahrmann, A.S. (2002). Diagnosis and treatment of movement impairment syndrome. St Louis, Missouri: Mosby Inc.

Shinkle, J., Nesser, T.W., Demchak, T.J., \& McMannus, D.M. (2012). Effect of core strength on the measure of power in the extremities. Journal of Strength and Conditioning Research, 26(2), 373-380.

Suter, E. \& Lindsay, D.M. (2001). Back muscle fatigability is associated with knee extensor inhibition in subjects with low back pain. Spine, 26(16), E361-E366.

Talukdar, K., Cronin, J., Zois, J., \& Sharp, A.P. (2015). The role of rotational mobility and power on throwing velocity. Journal of Strength and Conditioning Research, 29(4):905911.

Tihanyi, J., Apor, P., \& Fekete, G. (1982). Force-velocitypower characteristics and fiber composition in human knee extensor muscles. European Journal of Applied Physiology and Occupational Physiology, 48(3), 331-343.

Watkins, R.G., Uppal, G.S., Perry, J., Pink, M., \& Dinsay, J.M. (1996). Dynamic electromyographic analysis of trunk musculature in professional golfers. The American Journal of Sports Medicine, 24(4), 535-538.

Willson, J.D., Dougherty, C.P., Ireland, M.L., \& Davis, I.M. (2005). Core stability and its relationship to lower extremity function and injury. The Journal of the American Academy of Orthopaedic Surgeons, 13(5), 316-325.

Zemková, E., Cepková, A., Uvaček, M., \& Šooš, L. (2016). A novel method for assessing muscle power during the standing cable wood chop exercise. Journal of Strength and Conditioning Research [Epub ahead of print].

\section{E. Zemková}

Comenius University in Bratislava, Department of Sports Kinanthropology, Faculty of Physical Education and Sport, Nábr. arm. gen. L. Svobodu 9, 81469 Bratislava, Slovakia

e-mail: erika.zemkova@uniba.sk 
